\title{
An Auction-Based Incentive Mechanism for Non-Altruistic Cooperative ARQ via Spectrum-Leasing
}

\author{
Igor Stanojev ${ }^{1,2}$, Osvaldo Simeone ${ }^{2}$, Umberto Spagnolini ${ }^{1}$, Yeheskel Bar-Ness and Raymond L. Pickholtz ${ }^{3}$ \\ ${ }^{1}$ Dipartimento di Elettronica e Informazione, Politecnico di Milano, Milano 20133, Italy \\ ${ }^{2}$ CWCSPR, New Jersey Institute of Technology, Newark, New Jersey 07102-1982, USA \\ ${ }^{3}$ Department of Electrical Engineering, The George Washington University, Washington D.C. 20052, USA
}

\begin{abstract}
We propose and analyze a novel decentralized mechanism that motivates otherwise non-cooperative stations to participate as relays in cooperative ARQ protocol. Cooperation incentive is provided by the possibility for the source to lease a portion of retransmission slot for the traffic of relaying terminals. To further leverage the opportunistic nature of cooperative ARQ and obtain a fully decentralized solution, the (motivated) relaying nodes compete for access to the retransmission slot by trying to make the best retransmission offer. Effective arbitration of cooperative retransmissions is performed using auction theory (bidding), with the source in the role of the auctioneer, the relaying nodes acting as the bidders and the (use of the) retransmission slot as the bidding article. It is noted that the proposed solution can be seen as a practical framework for the implementation of cognitive radio networks running according to the property-rights model (spectrum leasing). Numerical results and analysis confirm the efficient dynamic resource allocation property of the proposed scheme, revealing the relevant gains in terms of expected number of (re)transmissions required for successful data delivery for both the source (primary) and the cooperating (secondary) nodes.
\end{abstract}

\section{INTRODUCTION}

Cooperative Automatic Repeat ReQuest (ARQ) protocol [1] is a promising technique that provides a synergy between two important communication paradigms, cooperation [2] and opportunistic resource allocation [3]. In particular, a source-destination link applying this protocol might hand over possible retransmissions to one of the available neighboring terminals that were able to decode the original transmission; thus, the cooperative transmission is prescribed only if needed in an opportunistic fashion [1]. Despite this attractive property, implementation of cooperative ARQ protocols (and cooperative protocols in general [2]) is often debated [4], since the underlying assumption that relays unconditionally assist communications without any benefit, is unrealistic for regular (e.g., users') mobile stations.

Given the above, we alleviate the assumption that available relays are willing to assist the ongoing transmission in an altruistic fashion, and propose an incentive mechanism for cooperation based on spectrum leasing. Specifically, incentive for (otherwise non-cooperative) relaying nodes is given by the possibility for the source to lease a portion of the retransmission slots (or equivalently a number of subcarriers in an OFDM system) for the traffic of retransmitting relay terminal in exchange for cooperative retransmission. To further leverage gains from opportunistic transmission and obtain a fully decentralized solution, the relays (now motivated to cooperate) compete for the retransmission slot by trying to make the best retransmission offer (i.e., reliability), while simultaneously satisfying some reliability requirements for transmission of their own data in case of offer acceptance. Effective selection of relay retransmissions is performed using auction theory (bidding) [5], with the source in the role of the auctioneer, the relays acting as the bidders, the (use of the) retransmission slot as the bidding article and the retransmission reliability as the selection criteria. In particular, due to its many attractive properties (as discussed in Sec. II), we employ Vickrey (second-price) auction [6] to study the system performance.

Beside providing an attractive solution for the deployment of cooperative ARQ via spectrum leasing, the proposed solution can be conversely seen as a practical framework for the implementation of cognitive radio networks running according to property-rights model (spectrum leasing) [7]. In such networks, primary (licensed) users may lease portions of the licensed spectrum to secondary (unlicensed) users in exchange for some form of retribution. Here, the role of the primary node is played by the original source and that of the secondary by the relaying nodes. Moreover, retribution from secondary to primary nodes is in the form of cooperation to the primary transmission. This enables on-the-air decisions and avoids the regulatory issues or money transactions that commonly hinder the implementation of the spectrum leasing concept. The idea of spectrum leasing via cooperation was originally proposed in [8]. The solution therein requires a centralized decision process at the primary node based on global system information. The proposal in this current work can be seen as a further elaboration on the ideas of [8] to offer a fully distributed solutions by exploiting cooperative ARQ and auction theory.

\section{VICKREY AUCTION}

Auctions are a widely accepted mechanism for distribution of a limited amount of resources among competing users [5]. 
In this section we present some background information regarding Vickrey auction, which will be useful in the following. It is noted that the considered framework is, however, not limited to Vickrey auctions and may be implemented via other auction types [9].

Among various auction types, the sealed bid second-price Vickrey auction is of particular interest, due to its attractive properties [6]. In this type of auction, the bidding item is awarded to the highest bidder at the price of the second highest bid. The most attractive property of Vickrey auction is its 'truth telling nature': a weakly dominated strategy for each bidder is to report their evaluations of the bidding item truthfully. In particular, [6] defines truthful bidding as bidding with the price at which the bidder would be on the margin of indifference as to whether he obtains the article or not. Important consequence of this property is that the bidder requires no information on other bidders' strategies or their evaluations of the bidding item, as this knowledge would not impact its bidding strategy.

Since the price paid by the winning bidder is not larger than its item evaluation, the bidders are guaranteed a nonnegative profit. This creates a strong motivation for the users to take part in auction, which in turn increases the profit of the auctioneer. Furthermore, the auctioneer has the option of setting the lowest acceptable price (i.e., the reserve price) which reflects its own evaluation of the bidding item. By declaring a reserve price, the auctioneer is also guaranteed a nonnegative payoff. The desirable properties of Vickrey auction have already been exploited by the communication community in, e.g., [10].

\section{System Model}

Consider a scenario with source $\mathrm{S}$, access point $\mathrm{AP}$ and a set of $K$ available relaying stations $\left\{\mathrm{R}_{k}\right\}_{k=1}^{K}$ (see Fig. 1 with $K=2$ ). The source $\mathrm{S}$ maintains an error-free transmission towards the AP by means of a memoryless retransmission protocol (ARQ) with transmission rate (of the original transmission) $C_{0, S}[\mathrm{bit} / \mathrm{s} / \mathrm{Hz}]$. Each (re)transmitted packet occupies a time-slot of normalized duration $T=1$. The relaying stations $\left\{\mathrm{R}_{k}\right\}$, if granted access by the source, are interested in transmitting their data to the AP, under the constraint of minimum reliability $q_{k, \min }, k=1, \ldots, K$. Reliability is defined as the probability that transmission is successful (see Sec. IV for more details). The opportunity for the relays' transmission arises in the case of erroneous S-AP transmission: upon receiving a NACK (Negative Acknowledgement) message from $\mathrm{AP}$, in order to increase the retransmission reliability (and thus reduce the overall number of (re)transmissions), the source is willing to lease its retransmission slot, provided that the awarded $\mathrm{R}_{k}$, besides transmitting its own data, will also use it for the retransmission of source's message. More specifically, S awards the retransmission slot to the relay that (i) decoded original message from $\mathrm{S}$ and $(i i)$ offers to provide the largest message retransmission reliability, and particularly larger than the reliability achievable by $\mathrm{S}$ alone. The relays, on the other hand, compete for access by trying to make the best retransmission offer to the source, while simultaneously maximizing the reliability for transmission of their own data in

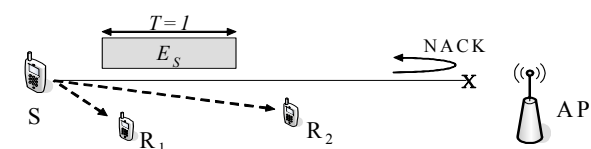

(a)

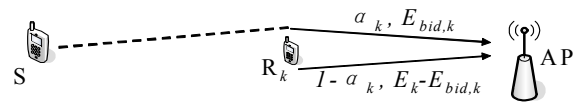

(b)

Fig. 1. Proposed retransmission model: (a) original source transmission (broadcast) with NACK from AP, (b) retransmission interval in case $\mathrm{R}_{k}$ wins the auction $(K=2)$.

case of the offer acceptance (under the constraint on $q_{k, \min }$ ). This interaction between the source and independent relays can be conveniently cast in the auction-theoretic framework, with the source in the role of the auctioneer, the relays as the bidders competing for access to retransmission slot, and the retransmission slot as the bidding article. In the following, we detail on the auction model for proposed cooperative ARQ scheme, applying the rules of Vickrey auction, as anticipated in Sec. II.

\section{A. Auction-Based Spectrum Leasing}

Upon receiving the NACK message from the AP (Fig. 1(a)), $\mathrm{S}$ determines the retransmission reliability $p_{\min }$ achievable with no help from the relays (as detailed in Sec. III-B and Sec. IV, the retransmission reliability $p_{\min }$ is estimated using a training sequence embedded in the NACK message), sets it as the reserve price and auctions the retransmission slot. During the original source transmission, the relays attempt to decode the message (Fig. 1-(a)) and, in case of successful decoding and retransmission request from the AP, calculate and submit the optimal bid for the auctioned retransmission slot. This is in the form of the source's packet retransmission reliability $p_{k}^{*}$, estimated from the training sequence in the NACK message (as detailed in Sec. IV). We assume, without loss of generality, that the relays that decoded the source message and are eligible for the auction are indexed as $\left\{\mathrm{R}_{k}\right\}_{k=1}^{n}$, where $n \leq K$ denotes the number of such stations. To simplify the model, once (any of) the relays decode and make the bid for a particular source message, the auction for this message, even in the case of its further retransmissions, is closed for other relays.

The process for calculating a bid is as follows. Each relay $\mathrm{R}_{k}$ has a target rate $C_{0, R_{k}}[\mathrm{bit} / \mathrm{s} / \mathrm{Hz}]$, a minimum required reliability $q_{k, \min }$ for transmission of its data and a per-slot available energy $E_{k}$ [Joule/channel symbol]. The profit for $\mathrm{R}_{k}$ is the difference between the reliability $q_{k}$ that it would achieve if winning the auction and $q_{k, \text { min }}$. Moreover, if the relay is not granted the access, the profit is zero. Reliability $q_{k}$ is calculated based on the outdated channel information at $\mathrm{R}_{k}$, obtained from the NACK message. In the case $\mathrm{R}_{k}$ 's offer is accepted, $\mathrm{R}_{k}$ sets a fraction $0<\alpha_{k}<1$ of the granted retransmission slot for the retransmission of the source message, and reserves the remaining fraction of $1-\alpha_{k}$ for transmission of its own data, as illustrated in Fig. 2-(a). 


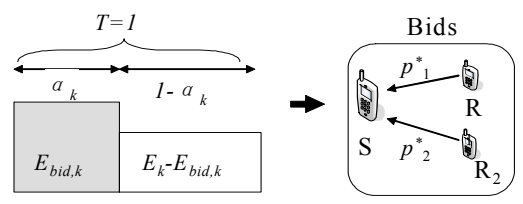

(a)

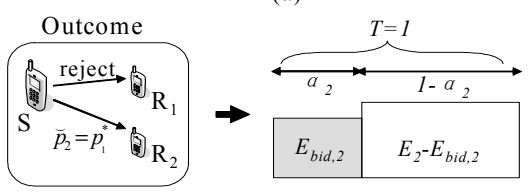

(b)

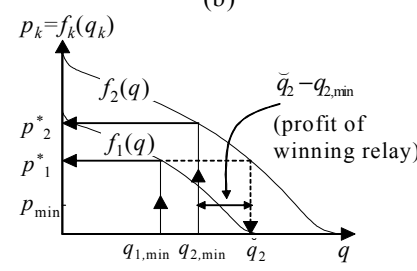

(c)

Fig. 2. Proposed auction-based model: (a) upon reception of a NACK, relays calculate and submit their bids, (b) auction outcome and (c) summary of the auction process with mapping $p_{k}^{*}=f_{k}\left(q_{k, \min }\right)$ and profit of the winning relay $\breve{q}_{\breve{k}}-q_{\breve{k}, \min }\left(K=n=2\right.$ and $p_{2}^{*}>p_{1}^{*}>p_{\min }$, with $\breve{k}=2$ and $\left.\breve{p}_{\breve{k}}=p_{1}^{*}\right)$.

Furthermore, the transmission energy invested for the source message retransmission (during the fraction $\alpha_{k}$ ) is $E_{b i d, k}<$ $E_{k}$ and thus, $E_{k}-E_{b i d, k}$ is the energy for transmission of its own data, during the slot fraction of duration $1-\alpha_{k}$. Following the principles of Vickrey auction, the optimal bid of the $k$ th relay reflects its evaluation of the item; herein, this boils down to the constraint $q_{k}=q_{k, \text { min }}$ which corresponds to the indifference evaluation for $\mathrm{R}_{k}$ between obtaining the retransmission slot or not. The optimization process behind selection of a bid $p_{k}$ is then:

$$
\begin{gathered}
p_{k}^{*}=\max _{\alpha_{k}, E_{b i d, k}} p_{k}\left(\alpha_{k}, E_{b i d, k}\right), \\
\text { s.t. } q_{k}\left(\alpha_{k}, E_{b i d, k}\right)=q_{k, \min }, \\
0<\alpha_{k}<1,0<E_{b i d, k}<E_{k} .
\end{gathered}
$$

Notice that the solution of optimization (1) can be regarded as a bid function, i.e., the mapping of the $k$ th bidder's evaluation of the item to its bidding offer, $p_{k}^{*}=f_{k}\left(q_{k, \min }\right)$. The underlying physical layer parameters defining the quantities in (1) are described in Sec. III-B, while the optimization process (1) is the focus of Sec. IV-A.

After the bids from (1) are calculated and submitted (Fig. 2-(a)), the source awards the auctioned slot to the relay $k$, where $\breve{k}=\arg \max _{k} p_{k}^{*}$, assuming that $p_{k}^{*} \geq p_{\min }$ (Fig. 2 -(b)); if $p_{k}^{*}<p_{\min }$, none of the relays is granted the access and the source performs retransmission on its own. The standard assumption, also adopted herein, is that, in the case of multiple equal (highest) offers, the situation is resolved by random allotment to one of the strongest bidders. Assuming that $p_{\vec{k}}^{*} \geq p_{\text {min }}$, i.e., that the auction resulted in the slot lease to $\mathrm{R}_{\breve{k}}$, the reliability required from $\mathrm{R}_{\breve{k}}$, according to Vickrey auction, is either the second highest bid or the reserve price $p_{\min }$, i.e., $\breve{p}_{\breve{k}}=\max \left\{p_{\min }, \max _{k \neq \breve{k}} p_{k}^{*}\right\}$ (for example in Fig. 2-(b), $K=n=2, \breve{k}=2, p_{\min }<p_{1}^{*}=\breve{p}_{2}<p_{2}^{*}$ ). Given the "price" $p_{\breve{k}}=\breve{p}_{\breve{k}}$, the relay $\mathrm{R}_{\breve{k}}$ can improve its profit $q_{\breve{k}}-q_{\breve{k} \text {,min }}$, with respect to the zero-profit ( $\left.q_{k}=q_{k, \min }\right)$ used to evaluate the bid (1). This leads to the following optimization problem to determine the relay's reliability $\breve{q}_{\breve{k}}$ and corresponding resource allocation $E_{b i d, \breve{k}}$ and $\alpha_{\breve{k}}$ :

$$
\begin{gathered}
\breve{q}_{\breve{k}}=\max q_{\breve{k}}\left(\alpha_{\breve{k}}, E_{b i d, \breve{k}}\right), \\
\text { s.t. } p_{\breve{k}}=\breve{p}_{\breve{k}}, \\
0<\alpha_{\breve{k}}<1,0<E_{b i d, \breve{k}}<E_{\breve{k}} .
\end{gathered}
$$

Notice that, while in (1) the focus is on bidding with the constraint on the reliability of relay $q_{k}=q_{k, \min }$, the optimization (2) is "dual" of (1), as here the constraint is the reliability of the source $p_{\breve{k}}=\breve{p}_{\breve{k}}$. Since the mapping $p_{k}=f_{k}(q)$ is unique for any value $q$, the auction process can be illustrated on Fig. 2-(c) where bidding reflects the mapping $q_{k, \min } \rightarrow p_{k}^{*}$, the value $\breve{p}_{\breve{k}}$ is the the agreed source reliability and mapping $\breve{p}_{\breve{k}} \rightarrow \breve{q}_{\breve{k}}>q_{\breve{k}, \min }$ corresponds to optimization (2).

\section{B. Physical Layer and Basic Definitions}

The channels over different links are modeled as independent complex Gaussian variables, invariant within the transmission slot. Delay between the (re)transmission slots is large enough to assume uncorrelated (block) fading. The following notation is employed to denote the instantaneous complex channel values within a (re)transmission slot: $h_{S}$ between $\mathrm{S}$ and $\mathrm{AP} ; h_{S R, k}$ between $\mathrm{S}$ and $\mathrm{R}_{k}$; and $h_{R S, k}$ between $\mathrm{R}_{k}$ and $\mathrm{AP}$, for any $k=1, \ldots, K$. The respective channel power gains are $g_{S}=E\left[\left|h_{S}\right|^{2}\right], g_{S R}=E\left[\left|h_{S R, k}\right|^{2}\right]$ and $g_{R S}=E\left[\left|h_{R S, k}\right|^{2}\right]$ (i.e., the channels have equal gain, for any $k=1, . ., K)$. The available energy per channel symbol of the source is denoted as $E_{S}$, energy of the $k$ th relay, as indicated above, is $E_{k}$, while the single-sided spectral density of the independent white Gaussian noise at the receivers is normalized to unity, $N_{0}=1$. As noted before, the target transmission rates of the (original transmission) of $\mathrm{S}$ and $\mathrm{R}_{k}$ are $C_{0, S}$ and $C_{0, R_{k}}$, respectively.

To determine the transmission reliability for a given (re)transmission slot, each transmitting node exploits the knowledge of the channel towards the AP, obtained via a training sequence embedded in the ACK/NACK message from AP (assuming time-division-duplex, TDD). Channel variation during the interval between the estimation instance and the consequent (re)transmission slot is accounted for and modeled through a temporal correlation parameter $\rho$, as in, e.g., [11]. Notice that delay between the downlink channel estimation and the following (re)transmission slot is not negligible but still much smaller than the delay between (re)transmissions, in order for block-fading to hold. The actual channel $h \in$ $\left\{h_{S}, h_{R S, 1}, . ., h_{R S, K}\right\}$ during the (re)transmission slot is outdated with respect to the channel at the receiving time interval 
$\hat{h}$ (with correlation $\rho$ ) and the corresponding modelling is [11]

$$
h=\rho \hat{h}+w,
$$

where $\hat{h} \sim \mathcal{C N}(0, g), g=E\left[|h|^{2}\right]$ and $w \sim \mathcal{C N}\left(0, \sigma^{2}\right)$ is the innovation term (due to the outdated knowledge) with variance $\sigma^{2}=\left(1-\rho^{2}\right) g$. The normalized power channel gain $|h|^{2} / \sigma^{2}$ takes the distribution of a noncentral chi-square variable with two degrees of freedom and noncentrality parameter $|\rho \hat{h}|^{2} / \sigma^{2}$, $|h|^{2} / \sigma^{2} \sim \chi_{2}^{2}\left(|\rho \hat{h}|^{2} / \sigma^{2}\right)$. From this distribution, the reliability can be easily evaluated by assuming coding at the Shannon limit for a given target rate and considering the outage probability, as detailed in the following section.

\section{System ANALYSIS}

This section provides the analysis of the model at hand. Firstly, we elaborate on the solutions of the optimization problems (1) and (2) that define the auction process. Then, we evaluate the system performance in terms of average number of (re)transmission slots required for reliable transmission of a source's packet; for analytical tractability, this task is performed assuming identical relays (parameters $q_{k, \min }, E_{k}$ and $C_{0, R_{k}}$ are equal for any $k$ ). Notice that the analysis of the average number of a relay's (re)transmissions is similar to that of the source and omitted here due to space limitations.

\section{A. Solving Problem (1)}

The optimization problem (1) provides the bid $p_{k}^{*}=$ $f_{k}\left(q_{k, \text { min }}\right)$ of relay $\mathrm{R}_{k}$. To elaborate, we start with the expressions for the reliabilities $p_{k}$ and $q_{k}$ of relay $\mathrm{R}_{k}$. These are defined as the probability of successful transmission, given the current information (3) available at $\mathrm{R}_{k}$ :

$$
\begin{aligned}
& p_{k}=\operatorname{Pr}\left\{C_{0, S} \leq C_{S, k} \mid \hat{h}_{R S, k}\right\} \\
& q_{k}=\operatorname{Pr}\left\{C_{0, R} \leq C_{R, k} \mid \hat{h}_{R S, k}\right\},
\end{aligned}
$$

where $C_{S, k}$ and $C_{R, k}$ are the rates achievable by $\mathrm{R}_{k}$ during the subslots of duration $\alpha_{k}$ and $1-\alpha_{k}$, respectively (we assume that "Gaussian codebooks" are used):

$$
\begin{aligned}
C_{S, k} & =\alpha_{k} \log _{2}\left(1+\left|h_{R S, k}\right|^{2} \frac{E_{b i d, k}}{\alpha_{k}}\right) \\
C_{R, k} & =\left(1-\alpha_{k}\right) \log _{2}\left(1+\left|h_{R S, k}\right|^{2} \frac{E_{k}-E_{b i d, k}}{1-\alpha_{k}}\right) .
\end{aligned}
$$

Applying constraint $q_{k}=q_{k, \min }$ of (1) to (4b) and (5b), we have:

$$
\begin{aligned}
& q_{k, \text { min }}=\operatorname{Pr}\left\{\left|h_{R S, k}\right|^{2} \geq \frac{\left(1-\alpha_{k}\right)\left(2^{C_{0, R_{k}} /\left(1-\alpha_{k}\right)}-1\right)}{E_{k}-E_{b i d, k}}\right\} \\
& =1-F_{\chi^{2}}\left\{\frac{\left(1-\alpha_{k}\right)\left(2^{C_{0, R_{k}} /\left(1-\alpha_{k}\right)}-1\right)}{\sigma_{R S, k}^{2}\left(E_{k}-E_{b i d, k}\right)}, \frac{\left|\rho \hat{h}_{R S, k}\right|^{2}}{\sigma_{R S, k}^{2}}\right\},
\end{aligned}
$$

where $F_{\chi^{2}}\{x, \mu\}$ is the cumulative distribution function (cdf) of the noncentral chi-square distribution with two degrees of freedom and noncentrality parameter $\mu$, taken at value $x$. We thus obtain from (6) the following relation between optimization parameters $\alpha_{k}$ and $E_{b i d . k}$ in (1) that satisfy $q_{k}=q_{k, \min }$ :

$$
\begin{array}{r}
E_{b i d, k}\left(\alpha_{k}\right)=E_{k}-\frac{\left(1-\alpha_{k}\right)\left(2^{C_{0, R_{k}} /\left(1-\alpha_{k}\right)}-1\right)}{\sigma_{R S, k}^{2} F_{\chi^{2}}^{-1}\left\{1-q_{k, \min },\left|\rho \hat{h}_{R S, k}\right|^{2} / \sigma_{R S, k}^{2}\right\}}, \\
0<\alpha_{k}<1, \quad 0<E_{b i d, k}<E_{k},
\end{array}
$$

where $F_{\chi^{2}}^{-1}\{x, \mu\}$ is the inverse of the cdf of the noncentral chi-square distribution with two degrees of freedom and noncentrality parameter $\mu$, taken at value $x$, i.e., $F_{\chi^{2}}^{-1}\left\{F_{\chi^{2}}\{x, \mu\}, \mu\right\}=x$.

Similarly to (6), exploiting (4a) and (5a), the source reliability $p_{k}$, which is the objective function in (1), becomes:

$$
\begin{aligned}
p_{k} & =\operatorname{Pr}\left\{\left|h_{R S, k}\right|^{2} \geq \frac{\alpha_{k}\left(2^{C_{0, S} / \alpha_{k}}-1\right)}{E_{b i d, k}\left(\alpha_{k}\right)}\right\} \\
& =1-F_{\chi^{2}}\left\{\frac{\alpha_{k}\left(2^{C_{0, S} / \alpha_{k}}-1\right)}{\sigma_{R S, k}^{2} E_{b i d, k}\left(\alpha_{k}\right)}, \frac{\left|\rho \hat{h}_{R S, k}\right|^{2}}{\sigma_{R S, k}^{2}}\right\},
\end{aligned}
$$

where the relation $E_{b i d, k}\left(\alpha_{k}\right)$ is given by (7). We can conclude that the optimization problem (1) boils down to the following one-dimensional problem for the determination of the time fraction:

$$
\alpha_{k}^{*}=\arg \max _{0<\alpha_{k}<1} \frac{\alpha_{k}\left(2^{C_{0, S} / \alpha_{k}}-1\right)}{\sigma_{R S, k}^{2} E_{b i d, k}\left(\alpha_{k}\right)}
$$

and the bidding value $p_{k}^{*}$ submitted to the source becomes:

$$
p_{k}^{*}=1-F_{\chi^{2}}\left\{\frac{\alpha_{k}^{*}\left(2^{C_{0, S} / \alpha_{k}^{*}}-1\right)}{\sigma_{R S, k}^{2} E_{b i d, k}\left(\alpha_{k^{*}}\right)}, \frac{\left|\rho \hat{h}_{R S, k}\right|^{2}}{\sigma_{R S, k}^{2}}\right\} .
$$

The above process, concluded with (10), describes the mapping of the $k$ th relay's minimum reliability, $q_{k \text {,min }}$, to the auction bid $p_{k}^{*}, p_{k}^{*}=f_{k}\left(q_{k, \min }\right)$ (notice that $q_{k, \min }$ is not shown explicitly in (10), but is an argument of $E_{b i d, k}\left(\alpha_{k}^{*}\right)$ from (7)).

\section{B. Solving Problem (2)}

The outcome of the auction in terms of the reliability achieved by the source can be written as

$$
\breve{p}=\max \left\{p_{\min }, \max _{k \neq \arg \max _{k} p_{k}^{*}} p_{k}^{*}\right\},
$$

where $k=1, . ., n$ and $p_{\min }$ stands for the reliability achieved by source alone:

$$
\begin{aligned}
p_{\min } & =\operatorname{Pr}\left\{C_{0, S} \leq C_{S} \mid \hat{h}_{S}\right\} \\
& =1-F_{\chi^{2}}\left\{\frac{2^{C_{0, S}}-1}{\sigma_{S}^{2} E_{S}}, \frac{\left|\rho \hat{h}_{S}\right|^{2}}{\sigma_{S}^{2}}\right\},
\end{aligned}
$$

where $C_{S}=\log _{2}\left(1+\left|h_{S}\right|^{2} E_{S}\right)$ is the rate achievable on the channel between the source and the access point.

Assuming that the auction resulted in a relay's access and that $\mathrm{R}_{\breve{k}}$ is the auction winner, i.e., $\breve{k}=\arg \max _{k} p_{k}^{*}$ and $p_{\breve{k}}^{*} \geq$ $p_{\text {min }}$, the second best offer that $\mathrm{R}_{\breve{k}}$ needs to provide to the source is $\breve{p}_{\breve{k}}=\breve{p}$, where $\breve{p}$ is given in (11). To map this price 
to the achievable reliability for its own transmission $\breve{q}_{\breve{k}}, \mathrm{R}_{\breve{k}}$ needs to perform optimization (2). As indicated in Sec. IIIA, this optimization problem is identical to (1) (namely, the parameters $\alpha_{k}$ and $E_{b i d, k}$ and the constraint $q_{k, \min }$ in (1) are substituted by $1-\alpha_{\breve{k}}, E_{\breve{k}}-E_{b i d, \breve{k}}$ and $\breve{p}_{\breve{k}}$ in (2), respectively) and the analysis in Sec. IV-A can be used for this task.

\section{Average Number of Transmission Slots (Source)}

In order to ease analysis of the average number of transmission slots required for successful decoding of the source message at the AP, in this subsection we assume relays with identical parameters $q_{k, \min }, E_{k}$ and $C_{0, R_{k}}$. With this assumption, it is justified to consider the number of relays participating in auction instead of considering the particular relays, and exploit the Markovian structure of the source message transmission process depicted in Fig. 3. There are $K+2$ states, with state $\mathcal{A}_{O R}$ standing for the original transmission of the source, state $\mathcal{A}_{0}$ denoting the retransmission of the source when no relays decoded its message, while states $\mathcal{A}_{n}, n=$ $1, ., K$, stand for the retransmissions when $n$ relays decoded the source message and participate in the auction. We recall that we are assuming memoryless ARQ (i.e., Type I) and that relaying users attempt to decode only original transmission and further retransmissions by the source (i.e., in states $\mathcal{A}_{O R}$ and $\mathcal{A}_{0}$ ) so that the same $n$ relays bid in all retransmissions of a given packet. It is easy to see that, apart from degenerate cases, the chain is irreducible and aperiodic and, thus, has a steadystate distribution. The steady-state probabilities for the states $\mathcal{A}_{O R}$ and $\mathcal{A}_{n}, n=0,1, \ldots, K$, are denoted as $\pi_{O R}$ and $\pi_{n}$, respectively. For the transition probabilities, we use notation $P_{S}^{(n)}$ for the average probability of successful (re)transmission (average reliability) when $n$ relays participate in auction, and notation $P_{d}^{(n)}$ for the probability that $n$ relays decoded the source transmission and are eligible to participate in auction.

The average number of transmissions for the source, $E\left[T_{S}\right]$, is the expected return time for the state $\mathcal{A}_{O R}$, which is given by:

$$
E\left[T_{S}\right]=1 / \pi_{O R}
$$

To determine $\pi_{O R}$, let us write the system of equilibrium equations for Markov process in Fig. 3:

$$
\begin{aligned}
1 & =\sum_{n=0}^{K} \pi_{n}+\pi_{O R} \\
\pi_{0} & =\left(\pi_{O R}+\pi_{0}\right)\left(1-P_{S}^{(0)}\right) P_{d}^{(0)} \\
\pi_{n} & =\left(\pi_{O R}+\pi_{0}\right)\left(1-P_{S}^{(0)}\right) P_{d}^{(n)}+\pi_{n}\left(1-P_{S}^{(n)}\right),
\end{aligned}
$$

for $n=1, . ., K$. This set is easily solved for $\pi_{O R}$ as:

$$
\pi_{O R}=\frac{1-\left(1-P_{S}^{(0)}\right) P_{d}^{(0)}}{1+\left(1-P_{S}^{(0)}\right) \sum_{n=1}^{K} P_{d}^{(n)} / P_{S}^{(n)}} .
$$

Thus, to find $E\left[T_{S}\right]$, we need to solve for $P_{S}^{(n)}$ and $P_{d}^{(n)}$.

1) Calculation of $P_{d}^{(n)}$ : The probability that $n$ out of $K$ relays decoded the source message, $P_{d}^{(n)}$, can be conveniently represented through binomial distribution:

$$
P_{d}^{(n)}=\left(\begin{array}{c}
K \\
n
\end{array}\right)\left(P_{d e c}\right)^{n}\left(1-P_{d e c}\right)^{K-n},
$$

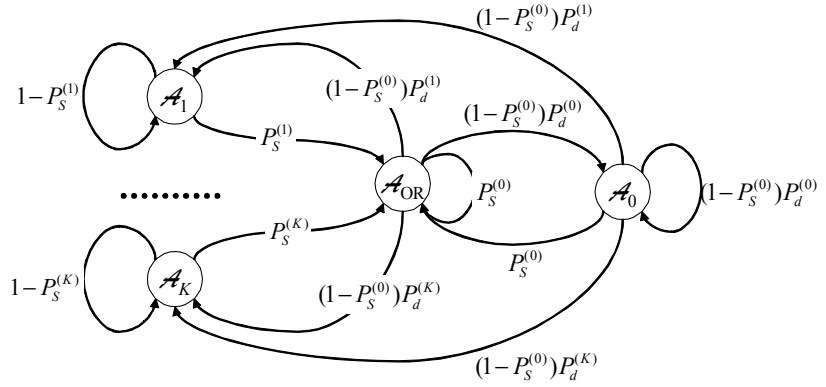

Fig. 3. Markov process for the source message transmission.

where $P_{d e c}$ is the probability of successful decoding at a relay, i.e., the probability that the achievable rate between the source and a relay, $C_{S R, k}=\log _{2}\left(1+\left|h_{S R, k}\right|^{2} E_{S}\right)$, for any $k=$ $1, . ., K$, is large enough to accommodate the transmission rate $C_{0, S}$ used by the source:

$$
P_{d e c}=\operatorname{Pr}\left\{C_{0, S} \leq C_{S R, k}\right\}=\exp \left(-\frac{2^{C_{0, S}}-1}{g_{S R} E_{S}}\right) .
$$

where we used the fact that $\left|h_{S R, k}\right|^{2} / g_{S R}$ is an exponentially distributed variable with rate parameter $\lambda=1$.

2) Calculation of $P_{S}^{(n)}$ : The probability $P_{S}^{(0)}$ is the average reliability of the source transmission, and can be obtained by averaging (numerically) (12) over the distribution of $\left|\hat{h}_{S}\right|$, which is Rayleigh:

$$
f\left(\left|\hat{h}_{S}\right|\right)=2\left|\hat{h}_{S}\right| / g_{S} \cdot \exp \left(-\left|\hat{h}_{S}\right|^{2} / g_{S}\right) .
$$

For $n=1,2, . ., K$ we instead average (numerically) (11) over both the distribution of $\left|\hat{h}_{S}\right|$ (as in (18)) and $\left|\hat{h}_{R S, k}\right|, k=$ $1, . ., n$, with

$$
f\left(\left|\hat{h}_{R S, k}\right|\right)=2\left|\hat{h}_{R S, k}\right| / g_{R S} \cdot \exp \left(-\left|\hat{h}_{R S, k}\right|^{2} / g_{R S}\right) .
$$

\section{Numerical Results}

Parameters employed throughout this section are: $N_{0}=1$, $E_{S}=E_{k}=1, C_{0, S}=C_{0, R_{k}}=1[\mathrm{bit} / \mathrm{s} / \mathrm{Hz}]$ and $q_{k, \min }=0$ (i.e., the relaying users transmit on a best-effort basis), for any $k=1, . ., K$. Fig. 4 focuses on a single relay to show the results of the optimization process (1), i.e., the mapping $p_{k}^{*}=$ $f_{k}\left(q_{k, \text { min }}\right)$, for $g_{R S}=7 \mathrm{~dB}$ and $\left|\hat{h}_{R S}\right|=1,3$, with different values of correlation parameter $\rho$. As expected, smaller values of $q_{k, \text { min }}$ enable the relay to use larger $E_{b i d, k}^{*}$ and $\alpha_{k}^{*}$ (not shown here) and therefore to bid with larger $p_{k}^{*}$. Notice that for $\rho \rightarrow 0$, the channel $\left|\hat{h}_{R S}\right|$ is not relevant as the distribution of the actual channel (recall Sec. III-B) remains Rayleigh. For $\rho \rightarrow 1$, on the contrary, there is no uncertainty on whether or not the relay can support its transmission and source's retransmission, and the bid becomes $p_{k}^{*}=0$ or $p_{k}^{*}=1$, depending on the value of $\left|\hat{h}_{R S}\right|$. To support the source with large probabilities $p_{k}^{*}$, it is clearly beneficial to maintain large $\left|\hat{h}_{R S, k}\right|$ with a relatively large correlation parameter $\rho$.

Fig. 5 shows the average number of transmission slots for the source $E\left[T_{S}\right]$ (equation (13)) and the relay $E\left[T_{R}\right]$ 
(applying analysis similar as in Sec. IV-C, not shown here) versus the placement of the relays $d$, for $g_{S}=-7 \mathrm{~dB}$ (SAP link in deep shadowing), $q_{\min }=0$ and $K=1,2,4$, 8. A simple geometrical model is considered, with relays positioned at approximately the same normalized distance $0<d<1$ from the source and $1-d$ from the access point; applying a path loss model (shadowing is not considered), the average channel power gains read $g_{S R}=g_{S} d^{-\gamma}$ and $g_{R S}=g_{S}(1-d)^{-\gamma}$, where $\gamma=3$ is the path loss exponent. The figure shows that there is a significant source performance increase due to the slot auction. For example, the average number of transmissions can be reduced more than five times here (from $E\left[T_{S}\right] \approx 17$ with $K=1$ or $K=0$, to $E\left[T_{S}\right] \approx 3$ with $K=8$ ). Notice that if only one relay participates in the auction $(n=1)$, there is no benefit for the source as its reserve price would be at the same time the auction outcome, $\breve{p}=p_{\min }$. The benefits for the relaying users ${ }^{1}$ are also meaningful, as they can maintain transmission with $E\left[T_{R}\right] \approx 2$ (when $K=1$ ). With larger $K^{2}$, performance of each relaying node decreases as the competition is, clearly, beneficial only for the source. Notice that if the relays are placed far away from the access point, $d \rightarrow 0$, so that the channels gains $h_{R S, k}$ are relatively small, or far away from the source, $d \rightarrow 1$, so that they can hardly decode its message, the auction benefit for the source is reduced. A similar conclusion holds for the relays, although for $d \rightarrow 1$ they can still achieve a relatively good performance (due to the strong channel towards the AP). In particular, notice that the placement $d$ where the best source or relay performance is achieved (i.e., where $E\left[T_{S}\right]$ or $E\left[T_{R}\right]$ is minimized) increases with the number of relays $K$. This is the consequence of multiuser diversity, i.e., the fact that with the increase of $K$, the probability that at least one of the relays will decode (recall Sec. IV-C.1) also increases, providing it with an opportunity of exploiting large channel $\left|h_{R S, \breve{k}}\right|$ for transmission. We note that the similar behavior was also observed in [8].

\section{CONCLUding REMARKS}

In this work, we have proposed and studied an auctionbased mechanism that leverages cooperative ARQ to opportunistically re-assign retransmission slots and exploits spectrum leasing to incentivize cooperation. The scheme can be conversely seen as a promising solution for the implementation of property-rights cognitive radio networks. In fact, dynamic and opportunistic spectrum access can be obtained via only local interactions at the physical and medium access layer. Numerical results reveal that, with the proposed model, the relaying (secondary) users can achieve excellent performance, while significantly improving performance of the original source (primary) node.

\footnotetext{
${ }^{1}$ Notice that, unlike for the source, the number of relay's transmission slots accounts also for the slots when not transmitting at all, either due to the successfully delivered source message, unsuccessful decoding at the relay, or not making the best offer.

${ }^{2} E\left[T_{R}\right]$ is a per-user delay. If per-(relaying) network delay was considered, curves $E\left[T_{R}\right]$ would simply scale down with factor $K$.
}

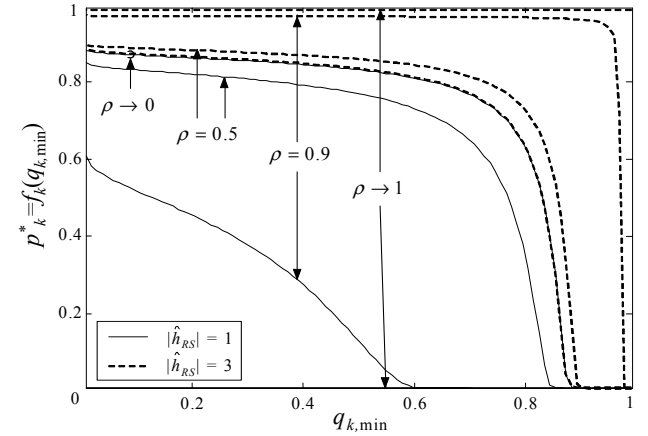

Fig. 4. Optimization (1): mapping $p_{k}^{*}=f_{k}\left(q_{k, \min }\right)$ for $\left|\hat{h}_{S P, k}\right|=1,3$ and varying values of correlation parameter $\rho$, for $g_{S P}=7 \mathrm{~dB}$.

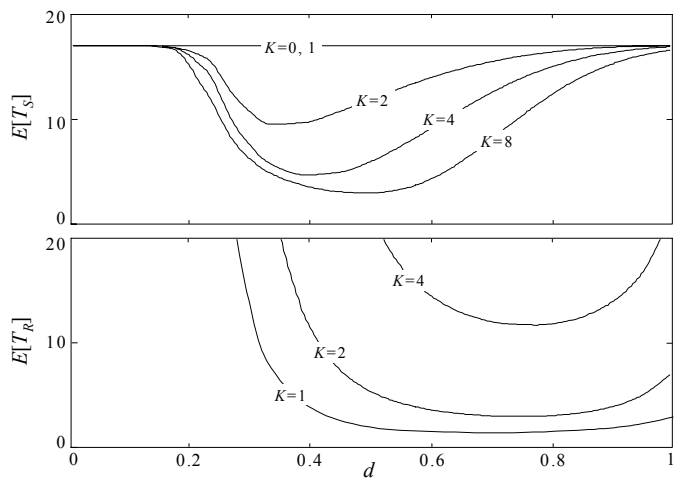

Fig. 5. Expected number of transmissions versus the placement of the relaying users $d$, for the source and a relay, $E\left[T_{S}\right]$ (13) (upper figure) and $E\left[T_{R}\right]$ (lower figure), respectively $\left(g_{S}=-7 \mathrm{~dB}, q_{\min }=0, K=1,2,4,8\right)$.

\section{REFERENCES}

[1] B. Zhao and M. C. Valenti, "Practical Relay Networks: A Generalization of Hybrid-ARQ," IEEE Journ. Selected Areas Commun., vol. 23, no. 1, pp. 7-18, Jan. 2005.

[2] A. Sendonaris, E. Erkip and B. Aazhang, "User cooperation diversityPart I: System description," IEEE Trans. Commun., vol. 51, pp. 19271938, Nov. 2003

[3] X. Liu, E. K. P. Chong, and N. B. Shroff, "Opportunistic transmission scheduling with resource-sharing constraints in wireless networks," IEEE Journ. Selected Areas Commun., vol. 19, no. 10, pp. 2053-2064, Oct. 2001.

[4] L. Lai and H. El Gamal, "On cooperation in energy efficient wireless networks: the role of altruistic nodes," IEEE Trans. Wireless Commun., vol. 7, no. 5, pp. 1868-1878, May 2008.

[5] P. Klemperer, "Auction Theory: A Guide to the Literature", J. Economics Surveys, vol. 13, no. 3, pp. 227-286, Jul. 1999.

[6] W. Vickrey, "Counterspeculations, Auctions, and Competitive Sealed Tenders", Journal of Finance, vol. 16, pp. 8-37, 1961.

[7] J. O. Neel, Analysis and Design of Cognitive Radio Networks and Distributed Radio Resource Management Algorithms, PhD. dissertation, Virginia Polytechnic Institute, September 2006.

[8] O. Simeone, I. Stanojev, S. Savazzi, Y. Bar-Ness, U. Spagnolini and R. Pickholtz, "Spectrum Leasing to Cooperating Secondary Ad Hoc Networks", IEEE J. Select. Areas Commun., vol. 26 no. 1, pp. 203-213, Jan. 2008 .

[9] I. Stanojev, O. Simeone, U. Spagnolini, Y. Bar-Ness and R. Pickholtz, "An Auction-Based Spectrum Leasing Approach to Cooperative ARQ", submitted to IEEE Trans. Commun.

[10] J. Sun, E. Modiano and L. Zheng, "Wireless Channel Allocation Using an Auction Algorithm," IEEE J. Select. Areas Commun., vol. 24, no. 5, pp. 1085-1096, May 2006.

[11] S. Ye, R. Blum, and L. Cimini, "Adaptive modulation for variable rate OFDM systems with imperfect channel information," in Proc. IEEE Vehicular Technology Conference, 2002. 